\title{
Dual Laser Switching for Dynamic Wavelength Operation in Amplified Optical Transmission
}

\author{
Shengxiang Zhu, Weiyang Mo, Daniel Kilper \\ Optical Science College, University of Arizona \\ szhu@optics.arizona.edu,wmo@optics.arizona.edu,dkilper@optics.arizona.edu
}

Aravind Padath Anthur, Liam Barry

School of Electronic Engineering, Dublin City University

aravind.anthur@dcu.ie,liam.barry@dcu.ie

\begin{abstract}
Fast switching of a dual laser PM-QPSK transceiver is used to mitigate channelpower excursions in amplified optical transmission under wavelength reconfiguration.

OCIS codes: $060.2330,060.1660,060.4264$.
\end{abstract}

\section{Introduction}

In today's optical networks, wavelength division multiplexed (WDM) channels are still largely provisioned as needed and remain fixed for the duration of their operating life. Migrating to a flexible optical physical layer in which the network can adapt in response to dynamic channel conditions and traffic requirements is a promising direction, which may lead to systems with tighter margins and greater resource efficiency [1]. While new software tools enable coordination of restoration between the IP and optical layers, systems are still limited by the slow response of the optical system control in large amplified networks. An unsolved problem in WDM networks for real-time reconfiguration is channel power dynamics that are generated during wavelength switching in optically amplified systems. Fast optical power transients associated with fiber breaks are largely solved by using fast feedforward pump control. However, even for transient controlled EDFAs, power excursions can still persist long after the transient has settled and require much slower wavelength selective switch and amplifier gain and tilt adjustments to resolve. Deviation from optimal power in optical networks can potentially result in sustained errors over long periods and also may interact with other power control mechanisms resulting in network instability. Positive power excursions beyond a predefined threshold can cause high nonlinear transmission impairments, and on the other hand, negative power excursions can result in excessive OSNR degradation, both of which degrade the network performance. Recent studies have looked at wavelength assignment algorithms based on the channel power interactions [2] and machine learning methods to select wavelengths based on historical performance information [3]. Fast laser source switching was shown to reduce and by duty cycle tuning remove power excursions [4]. In this paper, transmission of $100 \mathrm{~Gb} / \mathrm{s} \mathrm{PM-QPSK}$ signals using this fast laser switching technique by incorporating a laser pair at the source and local oscillator is studied through experiment and simulation. The dual laser switched pairs are used to cancel excursions for different pair wavelength locations in 8 spans transmission over $255 \mathrm{~km}$, representative of large metro scale networks. Using $230 \mathrm{~ns}$ guard bands which account for an $2.8 \%$ overhead, no impact on the bit error ratio is observed due to the wavelength switching. Experimental results are extended to a multi-hop case and show a reduction in the worst case excursion by half and to below $0.12 \mathrm{~dB}$ by duty cycle tuning.

\section{Principles of Dual Laser Switching}

In a dynamic network with wavelength switching, a change in input wavelength configuration can affect the gain of individual channels and generates power excursions. this effect has been thoroughly characterized for automatic gain controlled (AGC) amplifiers and shown to grow along an amplifier cascade while diminishing with larger channel loading, as wavelength dependent variations are averaged out [5]. Using two laser sources with an electro-optical switch to select between them within a single transceiver, a single channel power can be distributed over multiple wavelengths such that the excursion of these wavelengths can be reduced or canceled out [4]. When the switching period over these two wavelengths is faster than AGC control response time (on the order of $100 \mathrm{~s}$ or slower), there are no transient effects associated with this switching and such wavelength pairs can be provisioned without disturbing other channels in the system. 


\section{Experiment Setup}

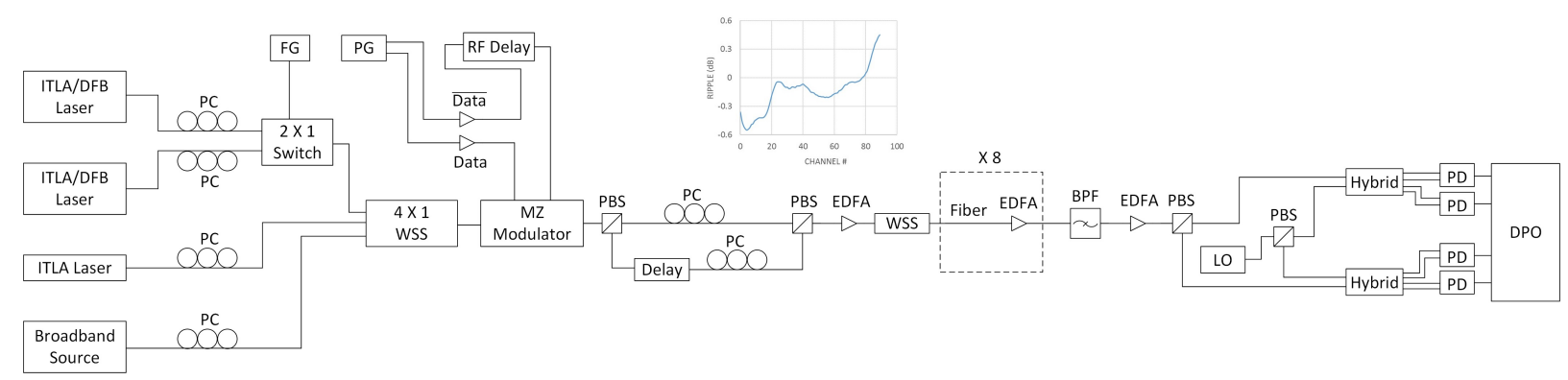

Fig. 1: 100Gbps PM-QPSK Experiment setup: A combination of two tunable Integrable Tunable Laser Assembly (ITLA) lasers and a 2x1 nanosecond speed switch is used to spread a 100G PM-QPSK signal over two arbitrary wavelengths and duty ratios. The inset shows ripple of a single EDFA. All EDFAs in the network have similar ripple function with $0.8 \mathrm{~dB}$ peak to peak.

The experimental setup is shown in Fig. 1. Two ITLA lasers are connected to a $2 \times 1$ electro-optic switch. The switching signal is combined with another ITLA laser and a broadband laser source by a 41 Wavelength Selective Switch (WSS) to support different channel configurations of the system. The combined signal is fed to an IQ modulator for 50 Gbps QPSK modulation which is driven by a 25 Gbaud PRBS11 pattern generator, where the I and Q signals are decorrelated by 53 symbols. A 100 Gbps PM-QPSK signal is generated by splitting and combing the signals with 258 symbols delay using two polarization beam splitters (PBS). The combined signal is then sent to an amplifier followed by a WSS to set channel launch power (instantaneous 'on' power rather than their average power) at $-3 \mathrm{dBm}$ into network. The network consists of 8 two-stage EDFAs operated at the gain of $18 \mathrm{~dB}$ and with $0.8 \mathrm{~dB}$ peak-topeak ripple (Fig. 1) with the total fiber length of $255 \mathrm{~km}$. A tunable bandpass filter is used to select a channel for BER analysis. The coherent receiver consists of an ITLA local oscillator (LO), optical hybrids and 4 balanced $28 \mathrm{GHz}$ photo detectors and a 4-channel digital oscilloscope (DPO). The off-line BER analysis program inside of DPO captures 1 million sample points during $20 \mathrm{~s}$ to make BER analysis for an existing channel. The receive side LO wavelength is tuned to one wavelength of the switching pair at a time for demodulation although in practice a second electro-optic switch would select between two pre-tuned LOs. Multiple switching period frames are captured (Fig. 2c) and BER analysis is made on each individual frame and averaged out to get the BER of the switching channels. In addition, by analyzing the bit errors on the rising and falling edge of a switching frame, the averaged transition time is $23 \mathrm{~ns}$, as is shown in Fig. 2d.

\section{Experiment Results}

In order to evaluate a worst case scenario for power excursions, a static channel is set up at wavelength 1548.57 $\mathrm{nm}$ (Channel 49) with a measured BER of 2.93E4. Two wavelengths of a switching pair are selected to be 1530.77 $\mathrm{nm}$ (Channel 4) and $1563.10 \mathrm{~nm}$ (Channel 85) according to the EDFA ripple. Fig. 2a shows the power after signal transmission and the peak and dip in each switching period result from propagation delay is measured to be $230 \mathrm{~ns}$. Combining this with the switching transition guard bands, the total required guard bands are corresponding to $276 \mathrm{~ns}$ $2.8 \%$ overhead or reduced data rate due to switching. Fig. $2 \mathrm{c}$ shows the signal power when the channel is switched and Fig. $2 d$ shows the BER for the same transient period with a transient time (BER from 0.5 to 1E-3) of 23ns. Fig. $2 \mathrm{~b}$ shows the change of BER of the existing channel (Ch.49) and one of the switching pair (Ch.85) with different duty ratios. With a duty ratio of $70 \%$, zero excursion is achieved on the existing channel, and therefore the BER keeps unchanged for Ch.49. Fig. 2e shows the change of OSNR and excursion in this case. Note that the BER decreases with positive excursion (Fig. 2b) in our experiment, but this is due to the fact that we are only transmitting a short distance of $255 \mathrm{~km}$ and the impact of nonlinearity is small. For other modulation formats, higher launch power or longer distances, positive power excursions will cause more nonlinear impairments. Fig. $2 \mathrm{~b}$ also shows that for the dual laser channel, the BER is stable and as good as that of existing channel. To study the effectiveness of switching in a network, different network channel loading configurations are measured. Fig. $2 \mathrm{f}$ shows the measured excursion on the existing channels by moving one of the switching pair from channel 85 to 55 while keeping another switching wavelength at channel 4. Three scenarios are compared. The blue diamonds are the measurements of static provisioning (no switching) with maximum of $-2 \mathrm{dBm}$ excursions observed. Orange squares show that the excursion goes down when the dual laser pair is used, with switching duty ratio of 0.5 . With fine tuning of duty ratio, zero excursion can be achieved as shown 


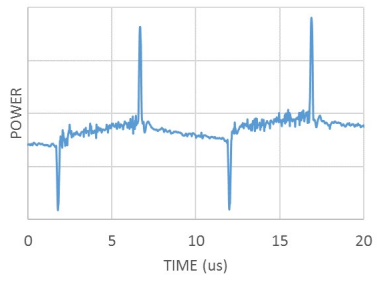

(a)

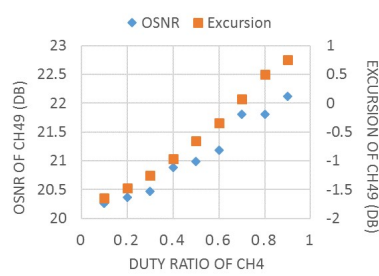

(e)

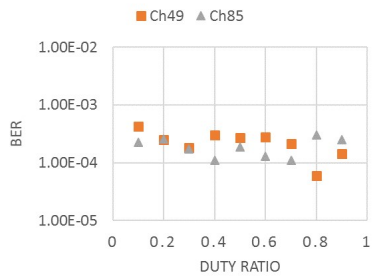

(b)

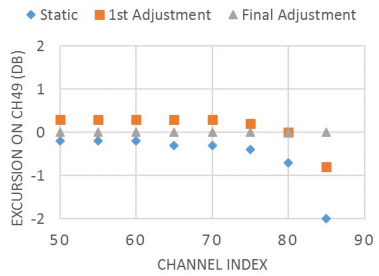

(f)

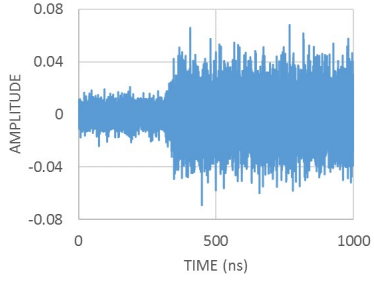

(c)

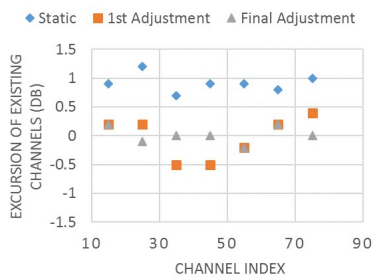

$(\mathrm{g})$

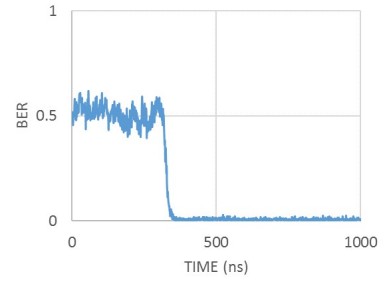

(d)

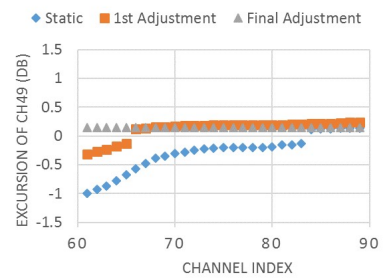

(h)

Fig. 2: Experiment Results: (a) Signal power after fiber transmission. (b) BER of existing channel with changed duty ratio of Ch.4. (c) Signal read from the DPO. (d) BER in transient state with time. (e) OSNR and excursion of Ch.49 with duty ratio of Ch.4. (f) Excursion of Ch.49 with different switching pair and its mitigation process. (g) Simulated excursion of Ch.49 with different switching pair and its mitigation process in a multi-hop network. (h) Excursions of different existing channels with switching pair of Ch.4 and Ch.85 and its mitigation process.

with grey triangles. We also explore the effectiveness of the switching technology by moving the original channel from channel 15 to 75 while keeping the switching wavelengths unchanged as shown in Fig. $2 \mathrm{~g}$. Minimal excursions are achieved with all different channel locations by adjusting switching duty ratio. We also study the effectiveness of the switching technology in a multi-hop network. Fig. 2h shows the simulation results of a 4-node network with different number of channels added/dropped on each span. We assume a new signal is needed from the first node through the network to the last node. In this case, excursions cannot be avoided with the new channel even using switching technology, however the maximum excursion can be largely minimized $(0.12 \mathrm{~dB}$ in Fig. $2 \mathrm{~h})$ by using a switching pair to mitigate the excursion of the span with fewest number of channels. On the other hand, a maximum power excursion of $-1 \mathrm{dBm}$ can occur with static provisioning. Additional studies of the wavelength blocking and other network considerations are still needed and will be the subject of future work.

\section{Conclusion}

We presented a fast switching technology for fast wavelength reconfiguration that not only mitigates channel power excursions to less than $+/-0.2 \mathrm{~dB}$, but also maintains channel OSNR unchanged and BER at the same level (under 1E-3). In the experiment, a single signal using a switching pair with $10 \mathrm{~s}$ period is switched into a $255 \mathrm{~km}$ network with 8 spans. By tuning the switching duty ratio and power, not only the BER of original channel is maintained during wavelength switching, but also the quality of the new switching signals using guard bands of $2.8 \%$ of the switching period.

This work was supported by the NSF Center for Integrated Access Networks (CIAN) EEC-0812072 and grant DESC0015867 through Advanced Scientific Computing Research within the Department of Energy Office of Science.

\section{References}

1. Kilper, Daniel C. Photonic Networks and Devices. Optical Society of America, 2015.

2. Ishii, Kiyo, Junya Kurumida, and Shu Namiki. IEEE Photonics Journal 8.1 (2016): 1-13.

3. Huang, Yisheng, et al., European Conference on Optical Communication (2016).

4. Ahsan, Atiyah S., et al. Journal of Optical Communications and Networking 7.9 (2015): 898-905.

5. Junio, Joseph, Daniel C. Kilper, and Vincent WS Chan. Journal of Optical Communications and Networking 4.9 (2012): A1-A7. 